\title{
A Numerical Study of Antisymmetric-Symmetric Mode of RB Convection through on Time Evolution
}

\author{
Fatima Zahra Benarab ${ }^{1}$, Rasikh Tariq ${ }^{2}$ \\ ${ }^{1}$ Laboratory of Mechanics, University Amar Telidji \\ Laghouat 03000, Algeria \\ ${ }^{2}$ Department of Mechanical Engineering, HITEC University \\ Khanpur Road, Taxila 47080, Pakistan \\ f.benarab@lagh-univ.dz; rasikh.tariq@hitecuni.edu.pk
}

\begin{abstract}
The purpose of this work is to study a specific case of Rayleigh-Benard convection. Convection undergoes transition timedependent regime on a sufficiently high Rayleigh Number. The critical Rayleigh value is calculated where antisymmetric-symmetric mode switches. This mode exhibits an odd number of rolls along $\mathrm{x}$ and an even number of cells in the perpendicular direction. It is clearly shown that this oscillatory convection is perfectly physical and periodic. Random probes are chosen to follow the timeevolution of the different parameters of the fluid such as dimensionless horizontal and vertical velocities, along with that temperature evolution is calculated too. These parameters are compared and discussed. Streamlines and Isotherm lines are presented at different times for one cycle of oscillation and are presented to show and understand the perfect mirror symmetry which is an important parameter of the studied case. The variation in Nusselt number is tracked from the hot and cold sides during time evolution. Nusselt Number curves show the intervals between the hot and cold flow in the same interval of values. The results shown in this paper are in good agreement with the precedent works. The study is done in a square cavity for a Rayleigh number varying between $10^{5}$ and $10^{6}$, the Prandtl number equal to 0.71 and a $10 \mathrm{~K}$ difference in the temperature. Governing equations of the phenomenon are numerically solved using FINITE VOLUME METHOD and the pressure-velocity coupling is matched by SIMPLER Algorithm.
\end{abstract}

Keywords: Rayleigh- Bénard, Thermal Convection, Bifurcation, Transitions, Oscillatory Mode, Mirror Symmetry

\section{Introduction}

The density of a fluid diminishes due to expansion upon heating. Therefore, hotter fluid has a tendency to elevate and cooler fluid to descent. The commencement of thermal convective motion occurs when the driving force (temperature difference) is enormous enough to overcome the dampening effects of thermal diffusivity and fluid viscosity. Above the commencement of convection, the fluid motion becomes turbulent as the velocity of the fluid increases with the temperature difference. Thermal convection is a key mechanism of heat transfer and is used to provide cooling for equipment in industrial processes [1]. Thermal convection is also abundant in nature. It plays part in the dynamics of the Earth's atmosphere [2] and in earth's mantle, as it is a vital cause for the motion of tectonic plates [3] .

Experimental studies of convection have often been carried out in Rayleigh-Bénard convection setup [4] , [5] . The RB convection system comprises of a closed cell of fluid which is heated from below and is cooled from above, RB convection is a classical problem in fluid dynamics having many issues of interest. It is vital for understanding stability of fluid flows and it is a worthy archetype to study pattern formation near the commencement of convection. Velocity and temperature display complex fluctuations with respect to time in turbulent regime [6]. These precise particulars of the fluctuations differ from flow to flow; however, statistical characteristics of these fluctuations are reproducible [7]. An important subject of curiosity is to illustrate and make sense of statistics of the fluctuations. [7]

Wang et al. [8] numerically studied thermal convection in a vertical cylindrical cavity heated from bottom, cooled from top and insulted from side walls. The height-to-radius ratio is fixed to unity whereas Prandtl number is changed from 0.04 to 1 and Rayleigh numbers ( $\mathrm{Ra}$ ) up to 16000 are deliberated. They find out that the transition from steady to oscillatory flow happens at lower Rayleigh number for small Prandtl number flow as compared to large Prandtl number flow. Stevens et al. [9] executed experimental measurements and numerical simulation on rotating RB convection having an aspect ratio changing from 0.5 to 2 . They observed; at fixed temperature difference between both plates, a sharp 
increase of heat transfer before the heat transfer drops drastically at stronger rotation rates. Torres et al. [10] solved the Navier-Stokes, continuity and energy equations in cylindrical coordinates using numerical scheme for rotating RB convection. Mizushima [11] studied thermal instability of a fluid in a rectangular box using a two dimensional flow field. Three boundary conditions are deliberated including stress free, perfectly insulating boundary conditions, and rigid/perfectly conducting. It was concluded that stability characteristics are very different between the cases of rigid and stress free side walls. Nandukumar et al. [12] investigated transition to chaos in RB convection of low Prandtl number fluids with free slip boundary conditions and performed direct three dimensional numerical simulation of the governing equations. Palymskiy [13] simulated RB convection using the Boussinesq model by solving the turbulent convectional flow of water and studied the time - space spectrums of kinetic energy (K.E.) temperature pulsations. Lappa [14] provided a comparative and critical review of secondary and oscillatory instabilities in thermal gravitational convection through examination of some existing studies, however in his recent works he [15] moved beyond the Boussinesq approximation; the resulting method allows the fluid flow properties to derive in a rational way by keeping a minimum empirical contamination.

Liu et al. [16] studied Rayleigh-Marangoni- Bénard convective instability in a two layer systems. A linear instability analysis and nonlinear instability analysis is performed (2D numerical simulation) to study the influence of thermocapillary force on the convective instability. The results show the strong effects of thermo-capillary force at the interface on the time-dependent oscillations at the onset of instability convection. Kolmychkov [17] presented a numerical simulation RB convection including a homogenous internal heat generation and investigated transitions between the conducting state, hexagonal, and roll flows for a Prandtl number range of 0.1-100 and Rayleigh number from subcritical values up to 1.5 times of its critical value. Ouertatani [18] applied finite volume method and full multi-grid acceleration on RB convection problem. Streamlines are also presented to analyses the natural convection flow patterns set up by buoyancy force. Shishkina et al.[19], shown the number required of nodes within each BL depends on $\mathrm{Nu}$ and $\mathrm{Pr}$ and grows with the Rayleigh number Ra for RB convection study.

The RB convection has large applications and a part of ongoing research. This research paper aims to study two dimensional RB convection setup by developing a mathematical model consisting of non-dimensional continuity, momentum and energy equation with the Boussinesq approximation, the Prandtl number equal to 0.71 and a $10 \mathrm{~K}$ difference in the temperature. The governing equations are to be solved by Finite Volume Method using FLUENT and solving pressure-velocity coupling using SIMPLER algorithm after setting a convergence criteria on a refined FLUENT mesh. This paper aims to calculate the Rayleigh critical value of the onset of antisymmetric-symmertic mode, and to develop an understanding of the time evolution of the flow parameters

\section{Mathematical Modelling}

The cavity which is heated from below and cooled from above corresponds to the configuration of the RayleighBénard dealing with the stability and motion of a fluid confined between two horizontal plates that are maintained at uniform temperatures, Figure 1.

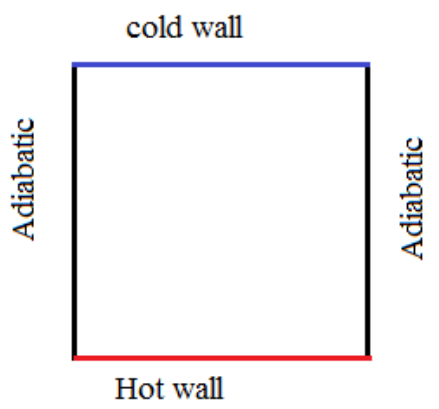

Fig. 1: Schematic of Rayleigh-Bénard configuration.

The mathematical model that governs such incompressible flow and satisfied the Boussinesq approximation consists of the dimensionless equations: 
Continuity Equation

$$
\frac{\partial U}{\partial X}+\frac{\partial V}{\partial Y}=0
$$

X-Direction Momentum Equation

$$
\frac{\partial U}{\partial \tau}+U \frac{\partial U}{\partial X}+V \frac{\partial U}{\partial Y}=-\frac{\partial P}{\partial X}+\left(\frac{\partial^{2} U}{\partial X^{2}}+\frac{\partial^{2} U}{\partial Y^{2}}\right)
$$

Y-Direction Momentum Equation

$$
\frac{\partial V}{\partial \tau}+U \frac{\partial V}{\partial X}+V \frac{\partial V}{\partial Y}=-\frac{\partial P}{\partial Y}+\frac{R a}{\operatorname{Pr}} \theta+\left(\frac{\partial^{2} V}{\partial X^{2}}+\frac{\partial^{2} V}{\partial Y^{2}}\right)
$$

Energy Equation

$$
\frac{\partial \theta}{\partial \tau}+U \frac{\partial \theta}{\partial X}+V \frac{\partial \theta}{\partial Y}=\frac{1}{\operatorname{Pr}}\left(\frac{\partial^{2} \theta}{\partial X^{2}}+\frac{\partial^{2} \theta}{\partial Y^{2}}\right)
$$

Equations (1-4) are converted into non-dimensional using following parameters.

$$
X=\frac{x}{H}, Y=\frac{y}{H}, U=\frac{u}{\alpha / H^{2}}, V=\frac{v}{\alpha / H^{2}}, P=\frac{p}{\rho(\alpha / H)^{2}}, \theta=\frac{T-T_{f}}{T_{C}-T_{f}}, S=\frac{t}{H^{2} / \alpha}
$$

The non-dimensional Raleigh Number and Prandtl Number are defined respectively by:

$$
R a=\frac{g \beta H^{3} \Delta T}{v \alpha} \text { and } \operatorname{Pr}=\frac{v}{\alpha}
$$

The initial and boundary conditions are given as follows:

$$
\begin{gathered}
\left\{\begin{array}{l}
Y=0 \\
Y=1
\end{array} \text { and } 0 \leq X \leq 1 ; U=V=0\right. \\
\text { For } Y=0 ; 0 \leq X \leq 1 ; \theta=\theta_{c} \\
\text { For } Y=1 ; 0 \leq X \leq 1 ; \theta=\theta_{f} \\
\left\{\begin{array}{l}
X=0 \text { and } 0 \leq Y \leq 1 ; U=V=0 \text { and } \frac{\partial \theta}{\partial X}=0 \\
X=1
\end{array}\right.
\end{gathered}
$$

Air is used at steady state, 1 atmospheric pressure and temperature equal to $305 \mathrm{~K}$ : 
Table 1: Physical Properties of air [20].

\begin{tabular}{|l|c|c|c|}
\hline \multicolumn{1}{|c|}{ Parameters } & Symbol & Value & Units \\
\hline Density & $\rho$ & 1.15 & $\mathrm{~kg} / \mathrm{m}^{3}$ \\
\hline Viscosity & $v$ & $1.86 \times 10^{-5}$ & $\mathrm{~kg} / \mathrm{m} . \mathrm{s}$ \\
\hline Specific heat & $\mathrm{C}_{\mathrm{p}}$ & 1006 & $\mathrm{~J} / \mathrm{kg} . \mathrm{K}$ \\
\hline Thermal conductivity & $\lambda$ & 0.0266 & $\mathrm{~W} / \mathrm{m} . \mathrm{K}$ \\
\hline Thermal diffusivity & $\alpha$ & $2.3 \times 10^{-5}$ & $\mathrm{~m}^{2} / \mathrm{s}$ \\
\hline Thermal expansion coefficient & $\beta$ & 0.00327 & $\mathrm{~K}^{-1}$ \\
\hline Prandtl number & $\mathrm{Pr}$ & 0.71 & - \\
\hline
\end{tabular}

\section{Numerical Simulation}

Equations (1-4) using with initial and boundary conditions (equations 5-8). are numerically solved by a finite-volume method [21] . ANSYS Fluent is used for numerical simulation. The Quick scheme is used for discretization of convective and diffusive terms. The pressure-velocity coupling is solved by SIMPLER algorithm. The obtained algebraic equations are solved by the line-by-line tri-diagonal matrix algorithm (TDMA). The convergence is declared when the maximum relative change between two consecutive iteration levels is less than $10-5$. A refined grid of $(20 \times 20)$ is used between the lower and upper wall, due to the existence of a strong temperature and velocity gradients near this walls, refining value is equal to 1.05. According to the work of Shishkina et al.[19] the chosen grids are estimate of a good quality .

\section{Results and Discussions}

According to linear theory, a significant change in convection will take place as soon as the critical Rayleigh number exceeds and Rayleigh-Bénard problem will become nonlinear [22] This kind of convection appears during the evolution from the stationary state to the fully developed turbulent regime, such a rich scenario of different structures and bifurcations that it is widely regarded as a reference problem for the study of different transition mechanisms in fluid dynamics[11] , [23] .From the previous work of Mizushima and Adachi [11], when the Rayleigh number sufficiently increased, convection can undergo transition to relatively complex and time-dependent regimes. An initial mode of convection can produce modes with other symmetries via a non-linear interaction mechanism. It's well known that on the onset of R-B convection the cavity contained one cell of streamlines; in the oscillatory mode, two cells appear inside the cavity, that can took two kinds of combination of possible symmetries[24] :

- (sa): The symmetric-antisymmetric mode. This mode is characterized by an even number of rolls along the horizontal direction and an odd number of vertices along the y direction;

- (as): The antisymmetric-symmetric mode. This mode exhibits an odd number of rolls along $\mathrm{x}$ and an even number of cells in the perpendicular direction;

Some steady and oscillatory 2D regimes for specific case is also considered in the study of Marcello Lappa [24] (cavity filled with a silicone oil with $\operatorname{Pr}=15$ ) They found that flow becomes oscillatory for $\operatorname{Ra}=5 \times 105$. Lappa [15] shows that when the behavior can be considered incompressible (very small value of the temperature gradient, $1 \mathrm{~K}$ only) and the average temperature is much smaller than the vibrational characteristic temperature, the emerging solution is very similar to that obtained by Goldhirsch et al. [25] . In this work even if the temperature gradient is equal to $10 \mathrm{~K}$, then the density does not undergo any significant change whereas specific heat remains constant in this interval. These conclusions, leads to find a good agreement of the results have shown here blow with those [11], [25], [26] .

\subsection{The Onset of the Antisymmetric-Symmetric Mode}

The second case of the oscillatory mode for a $\mathrm{Pr}=0.71$ i.e. antisymmetric-symmetric mode; is carefully discussed. In order to determine the beginning of the unsteady state, it's noticed that the flow becomes oscillatory beyond the value of Rac-os $2=4.25 \times 105$. This critical value is equal to 164.4 times the Rayleigh value of the onset of convection. To prove that the oscillations are perfectly physical and are not numerical instable, an arbitrary fix probe is chosen in the cavity with the same flow parameter is calculated for two time steps. 


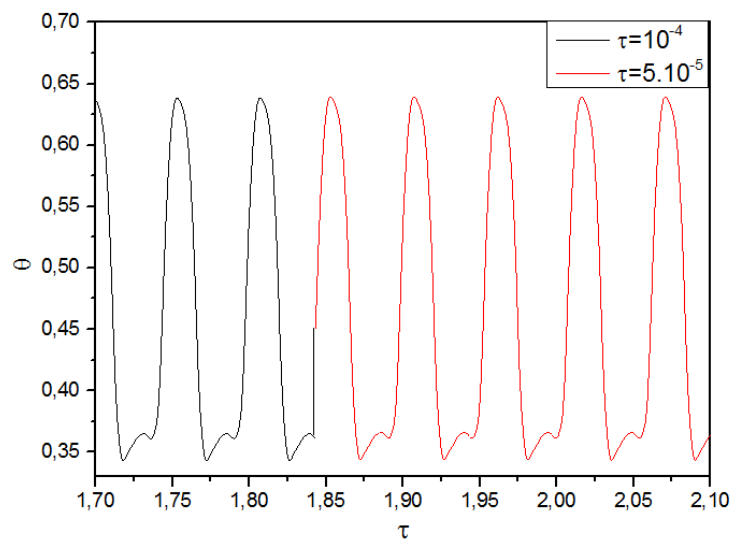

Fig. 2: Time dependent of vertical velocity component for two time step in P1 (choosing arbitrary) and for Rac-os2 $=4.25 \times 105$.

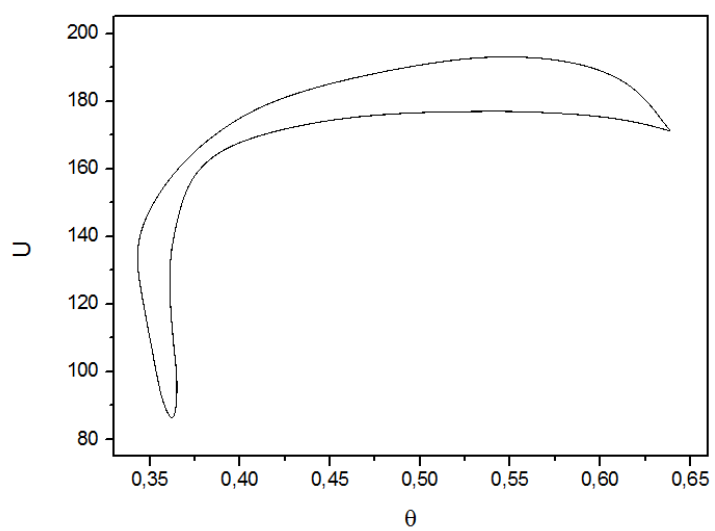

Fig. 3: Phase portrait of horizontal velocity component in function of temperature at P1 (choosing arbitrary), for Rac-os $2=4.25 \times 105$.

Figure 2 shows that there is no influence of the time step on the oscillation amplitude. Figure 3 represent the form portrait which is another test of oscillatory mode. It's clearly proven that this oscillatory are perfectly periodic.

\subsection{Time- Dependent Convection}

For a high Rayleigh number greater than 105 [25] a careful simulation is done for the evolution of the flow parameters. Chosen probes in the cavity are used to scan the field. Table 2 show the (x,y) position of each probe.

Table 2: Physical location of measurement probes.

\begin{tabular}{|c|c|c|}
\hline Probes & Dimensionless coordinate $\mathbf{X}$ & Dimensionless coordinate $\mathbf{Y}$ \\
\hline P1 & 0.5 & 0.5 \\
\hline P2 & 0.75 & 0.75 \\
\hline P3 & 0.75 & 0.25 \\
\hline P4 & 0.25 & 0.25 \\
\hline P5 & 0.25 & 0.75 \\
\hline
\end{tabular}

These probes are used to illustrate the temporal evolution of the horizontal, vertical velocity component and temperature; the results are shown in Figure 4. It is clear that these profiles are oscillatory and periodic, so the flow regime is unstable. It is observed that the amplitude of these oscillations changes from probe to another in the cavity. The difference in the degree of oscillation depends on the location of probes in relation to solid walls adiabatic to the hot wall and the cold wall. A decrease of the temperature can be seen from the bottom to the top; the time-evolution of temperature 
is shown in Figure 4A, a focus on the curves behavior can be divided on three groups, the first for the prob1 (in the middle) the second (probes 2-3) the third (probes 4-5) both of the last two groups follow the same variation. When one curves is increasing the other is decreasing with a little shift in period equal to the half of a period cycle, this time is the time of the heat propagation. The temperature variation in the center is the largest one with a mean value equal to 0.5 .

Figure 4B shows the variation on the vertical velocity in these case groups (probe 4-3), (probe 2-5) have the same behavior with 0.5 cycle shift between the two groups. Inside the group the value for example probes 3 and 4 . The velocity takes positive values in the side where the temperature growth. The second side where a decrease in temperature can be seen, the velocity has a negative vector. It's concluded that the probes took the same absolute values. For the central probe the velocity took a negative and positive values with the same absolute value throw the time evolution. For the horizontal velocity, in Figure 4C, the central probe took the largest values, they vary between negative and positive values with a half cycle shift. All this variation and difference between curves are the main results of temperature variation between the four sides of the cavity.

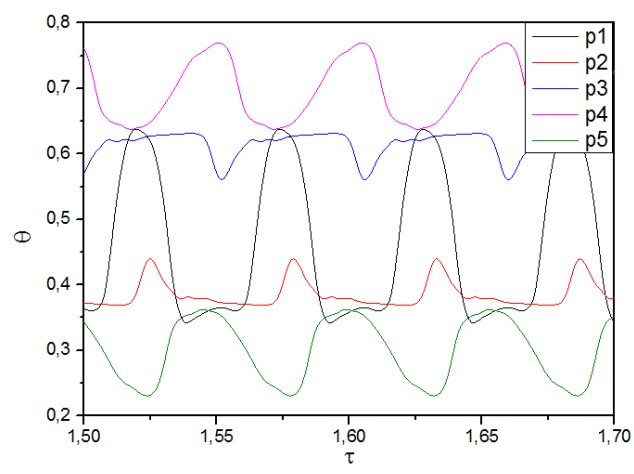

(A) : Time- evolution of the temperature

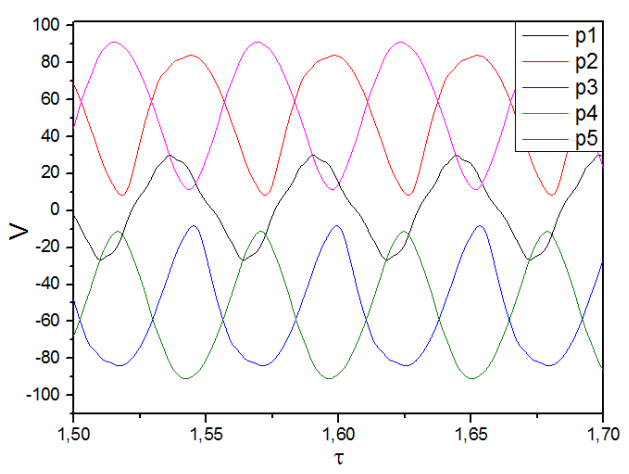

(C) :Time- evolution of the vertical velocity component

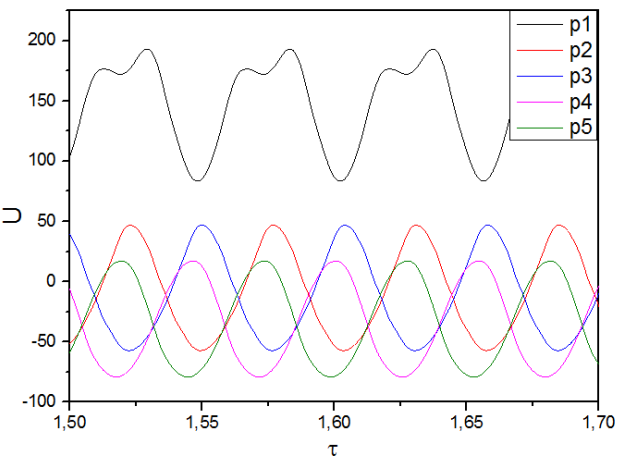

(B): Time- evolution of the horizontal velocity component

Fig. 4: Time-dependent of dimensionless horizontal (A)vertical velocity component (B) and dimensionless temperature , in probes (P1, $P 2, P 3, P 4, P 5$, ) for $R a=4.25 \times 10^{5}$.

Figure 5 shows a plot of Nusselt number (at the hot and cold line sides; bottom and top) as a function of the time. It can be seen that the two curves took the same values and reaches the same max and min values, with a shift equal to 0.03 which is the half of the total cycle time.

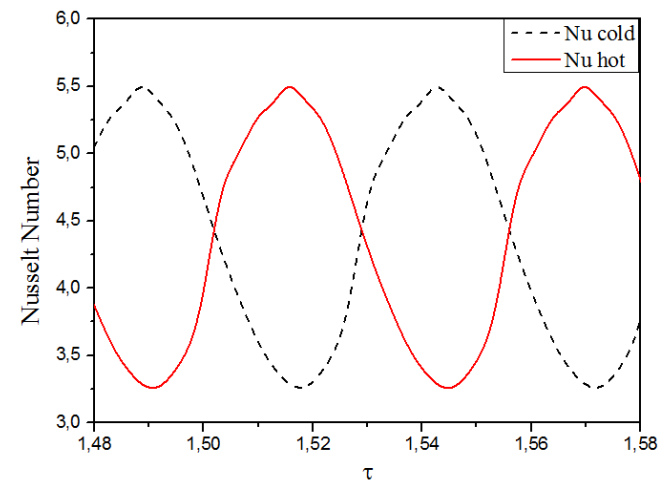

Fig. 5: The evolution of Nusselt number over time for both of hot and cold line sides. 


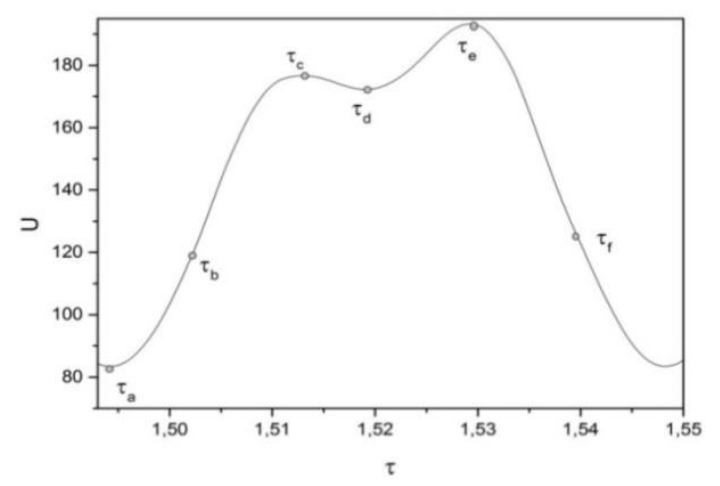

Fig. 6: Time-evolution of the vertical velocity component in $\tau=1$, for Rac-os $2=4.25 \times 10^{5}$.

\subsection{Time Historic Flow}

Figure 6 shows the instant where the isothermal lines are plotted. They are chosen following the growth of the curve, as it is one cycle of the time evolution of the vertical velocity. This section shows streamline and isotherm for different plots describing the dynamic at different chosen time instant in one full cycle (1.49-1.55). A full cycle of the fluid motion consists of several stages [25] .. The velocity decreases in the lower left hand corner, it increases in the upper right side. A new jet comes in the right hand corner the velocity increase rapidly with a decrease of velocity on the top, in the next three times.

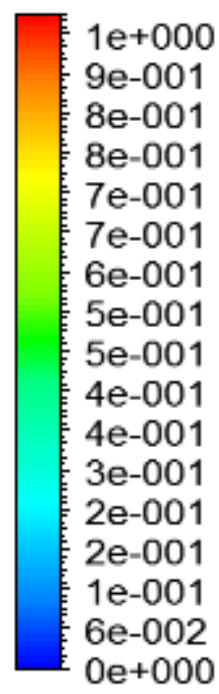

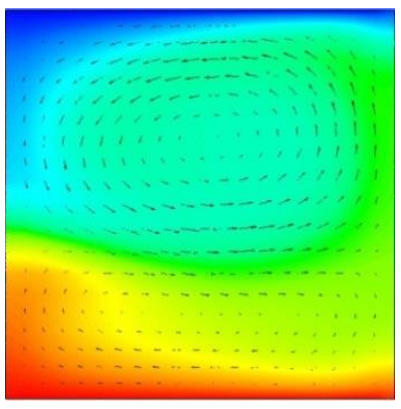

$\mathrm{a}-\tau_{\mathrm{a}}$

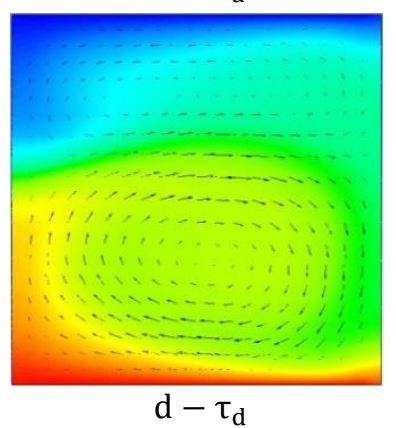

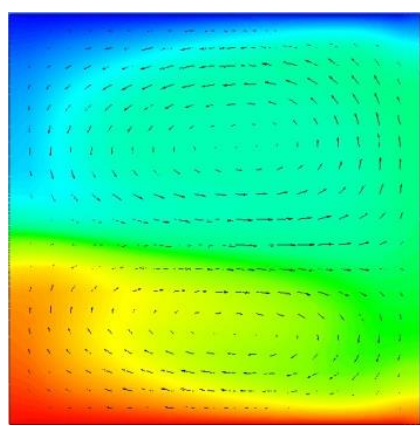

$b-\tau_{b}$

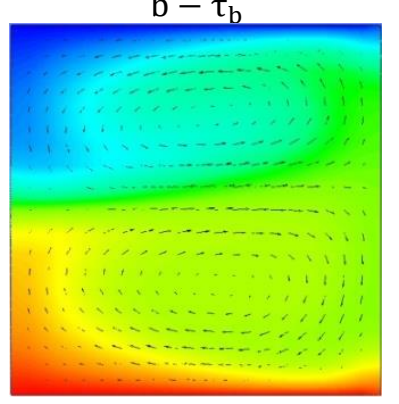

$\mathrm{e}-\tau_{\mathrm{e}}$

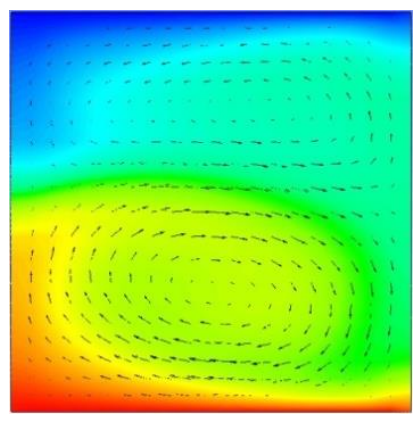

$c-\tau_{c}$

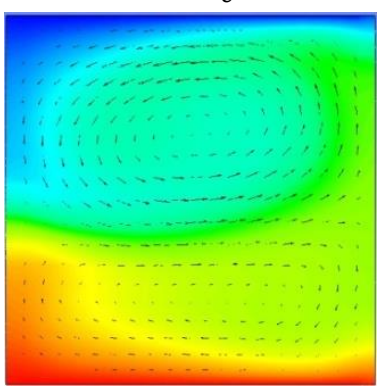

$f-\tau_{f}$

Fig. 7: Temperature distribution and velocity field of oscillatory convection Rayleigh for Rac-os $2=4.25 \times 10^{5}$.

In the fifth time it can be seen that the cavity perfectly divided in two parts with a horizontal line; this is called the mirror effect [25] , [26] R-B convection presents the perfect mirror symmetry with respect to the midsection. In this case the velocities of the cells take the same values with a difference in the direction. The upper roll takes a size much larger than that of the roll affecting the lower half of the enclosure. The latter, in turn, undergoes evident oscillatory motion, which seems to be produced by the periodic growth and decay of a secondary roll embedded in the lower circulation system (such interpretation being consistent with the clearly observable presence of a wave travelling along the bottom thermal boundary layer). 
In the last plot Figure 7f, the flow gets back to the first position growth of upper velocity and decreases in the lower side. The vector plots explain more the behavior of this evolution. As precisely described by [24] , [25] the system has three boundary layer, in the top, in the bottom and between the rolls.

When a cell roll get growing in size then the temperature circulation becomes larger inside of it. For example, the upper roll gets growing until it approaches the hottest side of the cavity. The temperature affects the flow direction (velocity vectors direction), than one roll start getting small and the other rise in size. Figure 7a show the lowest position of inflection here the upper cell is the largest, from another hand Figure $7 \mathrm{~b}$ is the one for the highest inflection position. The other plots show the transition between the inflection positions. A focus on Figure 7c and Figure 7e reveals that they led to some similarity between them in the cell size and where the mirror effect appear. In Figure $7 \mathrm{~d}$ the lower cell reached a level where the cold temperature at the top of the cavity affects so she starts to decrease, with an increase in the cold cell in parallel.

The physical reason is, that the perturbation in the velocity comes from the growth of temperature and have an influence on the flow by the way of buoyancy force, which will be mixed and dissipated in the center of the cavity creating a new decreases of velocity. This result is enhanced by the discussion in previous section where it is found that the comportment of the probes can be divided into three groups, one in the middle and two others in the high and lowest part of the cavity.

\section{Conclusion}

$\mathrm{RB}$ convection is a classical problem of Fluid Mechanics having many existing applications and still a vast knowledge to explore, therefore, this problem is still under research. In this research paper, RB convection problem is solved numerically using FLUENT (ANSYS Inc.) and following conclusion is obtained:

- The critical value of Rayleigh number is calculated for the onset of antisymmetric-symmertic mode.

- A perfectly physical and periodic mode is observed for oscillatory convection. Perfect mirror symmetry is observed by the time evolution analysis of velocities and temperature curves.

- The physical reason helps to understand the behavior of temperature change that affects directly the flow circulation and the variation of rolls size and circulation.

The results come up with a good agreement with proceeding work. Remind that the Prandtl number is equal to 0.71 and a $10 \mathrm{~K}$ is taken as temperature difference, in this interval the specific heat stays constant; density does not undergo big changes.

\section{Nomenclature}

$\mathrm{A}=$ Aspect Ratio $(\mathrm{A}=\mathrm{L} / \mathrm{H})[-]$

$\mathrm{H}=$ Cavity height $[\mathrm{m}]$.

$\mathrm{L}=$ Width of the cavity $[\mathrm{m}]$.

$\mathrm{X}, \mathrm{Y}=$ Dimensionless coordinates [-].

$\mathrm{P}=$ Dimensionless pressure [-].

$\mathrm{U}, \mathrm{V}=$ Components of dimensionless velocity [-].

$\theta=$ Dimensionless temperature [-].

$\mathrm{S}=$ Dimensionless time [-]

$\mathrm{Nu}=$ Number of Average Nusselt [-]

$\mathrm{g}=$ Gravity acceleration [m2 .s-1 ]

$v=$ Kinematic viscosity [m2 .s-1 ]

$\lambda=$ Thermal conductivity [W. m-1 .K-1 ]

$\rho=$ Density $[\mathrm{kg} \cdot \mathrm{m}-3]$

$\beta=$ Thermal expansion coefficient [K-1 ]

$\mathrm{TC}=$ Temperature of the hot wall $[\mathrm{K}]$.

$\mathrm{Tf}=$ Temperature of the cold wall $[\mathrm{K}]$.

$\mathrm{Ra}=$ Rayleigh Number [-].

$\operatorname{Pr}=$ Prandtl Number [-]. 


\section{References}

[1] W. M. Stacey. Fusion: An Introduction to the Physics and Technology of Magnetic Confinement Fusion, Second Edition, Wiley-Vch, 2010.

[2] E. van Doorn, B. Dhruva, K. R. Sreenivasan, and V. Cassella, "Statistics of wind direction and its increments," Phys. Fluids, vol. 12, no. 6, pp. 1529, 2000.

[3] P. Cardin and P. Olson, "Chaotic thermal convection in a rapidly rotating spherical shell: consequences for flow in the outer core," Phys. Earth Planet. Inter., vol. 82, no. 3-4, pp. 235-259, 1994.

[4] B. Henri, "Les tourbillons cellulaires dans une nappe liquide. - M'ethodes optiques d'observation et d'enregistrement," J. Phys. Theor. Appl., pp. 254-266, 1901.

[5] M. C. Cross, P. C. Hohenberg, and S. A. Safran, "Wave number selection in Rayleigh-Bénard convection: A numerical study," Phys. D Nonlinear Phenom., vol. 5, no. 1, pp. 75-82, 1982.

[6] E. S. C. Ching. Statistics and Scaling in Turbulent Rayleigh-Bénard Convection, Singapore: Springer, 2014.

[7] A. V. Getling, Rayleigh-Bénard Convection: Structures and Dynamics, Singapore: World Scie., 1998.

[8] S.-N. Xia, Z.-H. Wan, S. Liu, Q. Wang, and D.-J. Sun, "Flow reversals in Rayleigh-Bénard convection with nonOberbeck-Boussinesq effects," J. Fluid Mech., vol. 798, no. 6, pp. 628-642, 2016.

[9] R. J. a M. Stevens, H. J. H. Clercx, and D. Lohse, "Heat Transport And Flow Structure In Rotating Rayleigh-Bénard Convection," Eur. J. Mech. B/Fluids, vol. 40, pp. 41-49, 2013.

[10] V. Á. González, A. Z. Cruz, C. E. Arjonilla, D. P. Zepeda, and C. S. Forgach, "Critical Phenomena of a Drop Through a Stratified Fluid," in Selected Topics of Computational and Experimental Fluid Mechanics. Environmental Science and Engineering, J. Klapp, G. Ruíz Chavarría, A. Medina Ovando, A. López Villa, L. Sigalotti, Ed. Cham: Springer, 2015, pp. 497-503.

[11] J. Mizushima and T. Adachi, "Structural stability of the pitchfork bifurcation of thermal convection in a rectangular cavity," J. Phys. Soc., vol. 64, no. 12, pp. 4670-4683, 1995.

[12] N. Yada, P. Kundu, S. Paul, and P. Pal, "Different routes to chaos in low Prandtl-number Rayleigh-Bénard convection," Int. J. Non. Linear. Mech., vol. 81, pp. 261-267, 2016.

[13] D. J. Bespalko, "Direct Numerical Simulation of Turbulent," vol. XXXIII, no. 12, pp. 23-26, 2006.

[14] M. Lappa, "Secondary and oscillatory gravitational instabilities in canonical three-dimensional models of crystal growth from the melt. Part 1: Rayleigh-Bénard systems," Comptes Rendus - Mec., vol. 335, no. 5-6, pp. 253-260, 2007.

[15] M. Lappa, "A mathematical and numerical framework for the analysis of compressible thermal convection in gases at very high temperatures," J. Comput. Phys., vol. 313, no. 8, pp. 687-712, 2016.

[16] Q. S. Liu, B. H. Zhou, R. Liu, H. Nguyen-Thi, and B. Billia, "Oscillatory instabilities of two-layer RayleighMarangoni-Benard convection," Acta Astronaut., vol. 59, no. 1-5, pp. 40-45, 2006.

[17] V. V. Kolmychkov, O. S. Mazhorova, O. V. Shcheritsa, "Numerical study of convection near the stability threshold in a square box with internal heat generation," Phys. Lett. Sect. A Gen. At. Solid State Phys., vol. 377, no. 34-36, pp. 2111-2117, 2013.

[18] N. Ouertatani, N. Ben Cheikh, B. Ben Beya, and T. Lili, "Numerical simulation of two-dimensional RayleighBénard convection in an enclosure," Comptes Rendus Mécanique, vol. 336, no. 5, pp. 464-470, 2008.

[19] O. Shishkina, R. Jam Stevens, S. Grossmann, and D. Lohse, "Boundary layer structure in turbulent thermal convection and its consequences for the required numerical resolution," New J. Phys., vol. 12, no. 7, 2010.

[20] I. Pop and D.B. Ingham, Convective Heat Transfer. Oxford: Pergamon Press, 2001.

[21] S. V Patankar, Numerical Heat Transfer and Fluid Flow., 1980, pp. 196.

[22] E. L. Koschmieder, Bénard Cells and Taylor Vortices. Cambridge University Press, 1993.

[23] J. Pallares, M. P. Arroyo, F. X. Grau, and F. Giralt, "Experimental laminar Rayleigh-Bénard convection in a cubical cavity at moderate Rayleigh and Prandtl numbers," Exp. Fluids, vol. 31, no. 2, pp. 208-218, 2001.

[24] M. Lappa, "Some considerations about the symmetry and evolution of chaotic Rayleigh-Bénard convection: The flywheel mechanism and the 'wind' of turbulence," Comptes Rendus - Mec., vol. 339, no. 9, pp. 563-572, 2011.

[25] I. Goldhirsch, R. B. Pelz, and S. a. Orszag, "Numerical Simulation of Thermal Convection In a Two-Dimensional Finite Box," J. Fluid Mech., vol. 199, no. 1, pp. 1-28, 1989.

[26] M. Lappa, "Rotating Thermal Flows in Natural and Industrial Processes," Rotating Therm. Flows Nat. Ind. Process., no. $08,2012$. 